\title{
The Zero-Undetected-Error Capacity Approaches the Sperner Capacity
}

\author{
Christoph Bunte, Amos Lapidoth, Fellow, IEEE, and Alex Samorodnitsky
}

\begin{abstract}
Ahlswede, Cai, and Zhang proved that, in the noisefree limit, the zero-undetected-error capacity is lower-bounded by the Sperner capacity of the channel graph, and they conjectured equality. Here we derive an upper bound that proves the conjecture.
\end{abstract}

Index Terms-Sperner capacity, zero-undetected-error capacity, directed graphs, discrete memoryless channels

\section{INTRODUCTION}

A ZERO-undetected-error decoder (z.u.e. decoder) declares that a message was transmitted only if it is the only message that could have produced the observed output. If the output could have been produced by two or more messages, it declares an erasure. Such a decoder thus never errs: it either produces the correct message or an erasure.

The zero-undetected-error capacity (z.u.e. capacity) $C_{0 \text {-u }}$ of a channel is the supremum of all rates that are achievable with a z.u.e. decoder in the sense that the probability of erasure tends to zero as the blocklength tends to infinity [1], [2]. (It does not matter whether we define $C_{0 \text {-u }}$ using an average or a maximal erasure probability criterion.) Restricting the decoding rule cannot help, so $C_{0-\mathrm{u}}$ never exceeds the Shannon capacity $C$.

Although partial results exist (see Section (II), the z.u.e. capacity of general discrete memoryless channels (DMCs) is still unknown. The focus of this paper is the z.u.e. capacity of nearly noise-free channels. More precisely, we focus on $\varepsilon$ noise channels, that is, DMCs whose input alphabet $\mathcal{X}$ is a subset of their output alphabet $\mathcal{Y}$ and whose transition law $W$ satisfies

$$
W(x \mid x) \geq 1-\varepsilon \quad \text { for all } x \in \mathcal{X} .
$$

Here and throughout we assume that $0 \leq \varepsilon<1$. For $\varepsilon$ noise channels we derive an upper bound on $C_{0-\mathrm{u}}$. We then apply this result to study the limit of $C_{0 \text {-u }}$ as $\varepsilon$ tends to zero. Ahlswede, Cai, and Zhang proved that this limit is lowerbounded by the Sperner capacity of a certain related graph, and they conjectured equality [2]. Our upper bound proves this conjecture.

The Sperner capacity is defined using graph-theoretic language in Section IV] Here we give an alternative characterization in terms of codes (see also [2]). For this we need some standard notation.

C. Bunte and A. Lapidoth are with the Signal and Information Processing Laboratory at ETH Zurich. E-mail: \{bunte,lapidoth@isi.ee.ethz.ch\}.

A. Samorodnitsky is with the Institute of Compute Science at The Hebrew University of Jerusalem. His work is partially supported by grants from BSF and ISF. E-mail: salex@cs.huji.ac.il

This paper was presented in part at the 2014 ISIT conference.
A DMC is specified by its transition law $W(y \mid x), x \in \mathcal{X}$, $y \in \mathcal{Y}$, where $\mathcal{X}$ and $\mathcal{Y}$ are finite input and output alphabets. Feeding a sequence of input symbols $\mathbf{x}=\left(x^{(1)}, \ldots, x^{(n)}\right)$ to a DMC of transition law $W$ produces a random sequence of output symbols $\mathbf{Y}=\left(Y^{(1)}, \ldots, Y^{(n)}\right)$ whose joint probability mass function (PMF) is

$$
W^{n}(\mathbf{y} \mid \mathbf{x}) \triangleq \prod_{1 \leq j \leq n} W\left(y^{(j)} \mid x^{(j)}\right), \quad \mathbf{y} \in \mathcal{Y}^{n}
$$

The support of $W$ is the set of all pairs $(x, y) \in \mathcal{X} \times \mathcal{Y}$ for which $W(y \mid x)$ is positive; it is denoted by $\mathfrak{S}(W)$. Similarly, if $P$ is a PMF on $\mathcal{X}$, then $\mathfrak{S}(P)$ denotes the set of all $x \in \mathcal{X}$ for which $P(x)$ is positive. We write $P W$ for the PMF on $\mathcal{Y}$ induced by $P$ and the channel $W$

$$
(P W)(y)=\sum_{x \in \mathcal{X}} P(x) W(y \mid x), \quad y \in \mathcal{Y} .
$$

If $A \subseteq \mathcal{X}$, then we write $P(A)$ in lieu of $\sum_{x \in A} P(x)$. The Cartesian product of two sets $A$ and $B$ is denoted by $A \times B$. The $n$-fold Cartesian product of $A$ with itself is denoted by $A^{n}$, and the cardinality of $A$ is denoted by $|A|$. All logarithms are natural $\log$ arithms, and we adopt the convention $0 \log \frac{1}{0}=0$.

We define a blocklength- $n$ Sperner code for a DMC $W$ with $\mathcal{X} \subseteq \mathcal{Y}$ and $W(x \mid x)>0$ for all $x \in \mathcal{X}$ as a collection of length- $n$ codewords $\mathbf{x}_{1}, \ldots, \mathbf{x}_{M}$ with the property

$$
W^{n}\left(\mathbf{x}_{m} \mid \mathbf{x}_{m^{\prime}}\right)=0 \quad \text { whenever } m \neq m^{\prime} .
$$

The rate of the code is $n^{-1} \log M$. The largest rate of a Sperner code is a function of the channel law $W$ and the blocklength $n$. In fact, it depends on $W$ only via its support $\mathfrak{S}(W)$. The supremum over $n$ of the largest rate of blocklength- $n$ Sperner codes is the Sperner capacity $C_{\mathrm{Sp}}$ of the channel.

We emphasize that a Sperner code is not a zero-error code. Indeed, the definition does not preclude the existence of a sequence $\mathbf{y} \in \mathcal{Y}^{n}$ such that $W^{n}\left(\mathbf{y} \mid \mathbf{x}_{m}\right)>0$ and $W^{n}\left(\mathbf{y} \mid \mathbf{x}_{m^{\prime}}\right)>0$ for some $m \neq m^{\prime}$. What cannot happen with a Sperner code is that one codeword is corrupted by the channel into another codeword. In other words, at the output we either receive the correct codeword or some sequence of output letters that is not a codeword.

In the notation above, Ahlswede, Cai, and Zhang proved in [2. Theorem 2] that for $\varepsilon$-noise channels,

$$
\varliminf_{\varepsilon \rightarrow 0} C_{0-\mathrm{u}} \geq C_{\mathrm{Sp}}
$$

and they conjectured equality. Our main result is the following inequality. 
Theorem I.1. For every $\varepsilon$-noise channel,

$$
C_{0 \text {-u }} \leq \log \left(e^{C_{\mathrm{Sp}}}+\varepsilon|\mathcal{X}|(|\mathcal{Y}|-1)\right) .
$$

Combining Theorem [.1] with (5) proves their conjecture:

Theorem I.2. For $\varepsilon$-noise channels,

$$
\lim _{\varepsilon \rightarrow 0} C_{0-\mathrm{u}}=C_{\mathrm{Sp}},
$$

where the limit is to be understood in a uniform sense with respect to all $\varepsilon$-noise channels with given $\mathfrak{S}(W)$.

A proof of Theorem I.1 is given in Section $\mathrm{V}$. Before providing an outline of this proof, we try to explain why Corollary I.2 is plausible. If we use a Sperner code in conjunction with a z.u.e. decoder, then an erasure can occur only if the codeword is corrupted, which happens with probability at most $1-(1-\varepsilon)^{n}$. This suggests that $C_{\mathrm{Sp}}$ should be a lower bound to $C_{0 \text {-u }}$ when $\varepsilon$ is very small (ignoring the issue that $n$ tends to infinity before $\varepsilon$ tends to zero). Conversely, any code whose maximal probability of erasure under z.u.e. decoding is smaller than $(1-\varepsilon)^{n}$ must be a Sperner code. Since for all rates strictly smaller than $C_{0 \text {-u }}$ the probability of erasure can be driven to zero exponentially fast (see below), this suggests that $C_{\mathrm{Sp}}$ should be an upper bound on $C_{0 \text {-u }}$ for small $\varepsilon$ (ignoring the issue that the exponent of the erasure probability may become arbitrarily small as $\varepsilon$ becomes small and the rate approaches $C_{0-\mathrm{u}}$ )

As to the outline of the proof of Theorem I.1, we first show that a multi-letter version of Forney's lower bound on $C_{0-\mathrm{u}}$ is asymptotically tight even when the input distributions are restricted to be uniform over their support (Section III). We then upper-bound the multi-letter expression using Jensen's inequality followed by algebraic manipulations that yield a still looser bound. Thanks to the input distribution being uniform, this looser bound depends only on $\varepsilon$ and the support of $W$. The final step is to use graph-theoretic techniques, which are introduced in Section IV] to obtain the desired upper bound. These techniques include upper-bounding a sum that depends only on the in-degrees of the vertices of a graph $G$ by the maximum size of any induced acyclic subgraph of $G$. They also include showing that the Sperner capacity of a graph $G$ can be expressed as the limit as $n$ tends to infinity of $1 / n$ times the logarithm of the maximum cardinality of any induced acyclic subgraph of the $n$-fold strong product of $G$ with itself.

\section{BACKGROUND}

To put our result into perspective, we briefly review some of the literature on the z.u.e. capacity and related concepts. We begin with Forney's lower bound

$$
C_{0 \text {-u }} \geq \max _{P} \sum_{y \in \mathcal{Y}}(P W)(y) \log \frac{1}{P(\mathcal{X}(y))},
$$

where the maximum is over all PMFs on the input alphabet $\mathcal{X}$, and where $\mathcal{X}(y)$ denotes the set of all $x \in \mathcal{X}$ for which $W(y \mid x)$ is positive [3]. His bound can be derived using standard random coding where each component of each codeword is drawn IID from a PMF $P$. In general, (8) is not tight 1 A tighter lower bound was derived in [1], [2], [4] using random coding over constant composition codes

$$
C_{0 \text {-u }} \geq \max _{P} \min _{\substack{V \ll W: \\ P V=P W}} I(P, V) .
$$

The minimum in (9) is over all auxiliary DMCs $V(y \mid x), x \in$ $\mathcal{X}, y \in \mathcal{Y}$ such that $V(y \mid x)=0$ whenever $W(y \mid x)=0$ (in short $V \ll W)$ and such that $V$ induces the same output distribution under $P$ as the true channel $W$.

Since any code for the product channel $W^{n}$ is also a code for the channel $W$ of $n$ times the blocklength and $1 / n$ times the rate, it follows that the bounds (8) and (9) can be improved by applying them to $W^{n}$ and normalizing the result by $1 / n$. For example, the $n$-letter version of (8) is

$$
C_{0-\mathrm{u}} \geq \frac{1}{n} \max _{P} \sum_{\mathbf{y} \in \mathcal{Y}^{n}}\left(P W^{n}\right)(\mathbf{y}) \log \frac{1}{P\left(\mathcal{X}^{n}(\mathbf{y})\right)},
$$

where the maximum is over all PMFs $P$ on $\mathcal{X}^{n}$, and where $\mathcal{X}^{n}(\mathbf{y})$ denotes the set of all $\mathbf{x} \in \mathcal{X}^{n}$ for which $W^{n}(\mathbf{y} \mid \mathbf{x})>0$. A numerical evaluation in [2] of the single-letter and two-letter versions of (9) for a particular channel suggests that a strict improvement is possible and hence that (9) is not always tight.

The $n$-letter version of (9) becomes tight as $n$ tends to infinity [1], [2]. Since the proof of (9) shows that for all rates less than the RHS of (9) the probability of erasure can be driven to zero exponentially fast, it follows that this is also true for all rates less than the $n$-letter version of the bound, and hence for all rates less than $C_{0-\mathrm{u}}$.

In Section III we prove that also the $n$-letter version of the weaker bound (8) is asymptotically tight, and that this is true even when the maximization is restricted to PMFs that are uniform over their support. This result will be crucial in the proof of Theorem I.1.

We have already pointed out that the Shannon capacity $C$ is an upper bound to $C_{0-\mathrm{u}}$. In fact, this bound is often tight. Indeed, Pinsker and Sheverdyaev [5] proved that $C_{0-\mathrm{u}}=C$ whenever the bipartite channel graph is acyclic. The bipartite channel graph is the undirected bipartite graph whose two independent sets of vertices are the input and output alphabets of the channel, and where there is an edge between an input $x$ and an output $y$ if $W(y \mid x)>0$ (it is customary to draw the inputs on the left and the outputs on the right and to label the edges with the transition probabilities). Acyclic means that we cannot find an integer $n \geq 2$, distinct inputs $x_{1}, \ldots, x_{n}$ and distinct outputs $y_{1}, \ldots, y_{n}$ such that $W\left(y_{j} \mid x_{j}\right)>0$ and $W\left(y_{j} \mid x_{j+1}\right)>0$ for all $j \in\{1, \ldots, n\}$ where $x_{n+1}=x_{1}$. Important examples of channels with acyclic bipartite channel graphs are the binary erasure channel and the Z-channel.

Csiszár and Narayan [1] proved that $C_{0 \text {-u }}=C$ whenever there exist positive functions $A$ and $B$ such that

$$
W(y \mid x)=A(x) B(y), \quad \text { whenever } W(y \mid x)>0 .
$$

They also proved that in this case (9) is tight. Their result is stronger than Pinsker-Sheverdyaev because every DMC with an acyclic bipartite channel graph possesses a factorization of its channel law in the sense of (11), but the converse is not true.

\footnotetext{
${ }^{1} \mathrm{An}$ example where it is not tight is the Z-channel [4].
} 


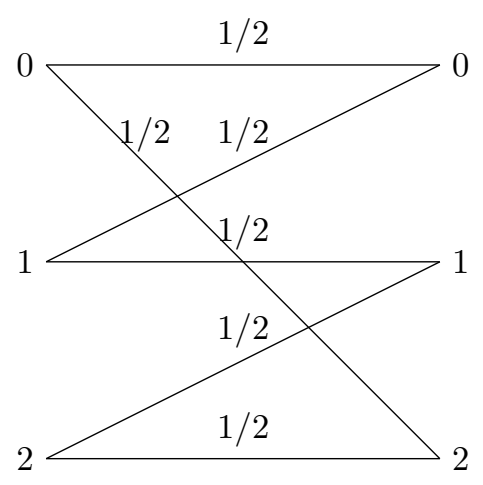

Fig. 1. The graph contains a cycle but the channel law factorizes.

For example, the graph shown in Figure 1 contains a cycle, yet the channel law factorizes with the choice $A=B=1 / \sqrt{2}$. In fact, they conjecture that a necessary condition for $C_{0-\mathrm{u}}=C$ is that (11) hold on some capacity-achieving subset of inputs (which is clearly also sufficient).

The fact that $C_{0-\mathrm{u}}=C$ for the Z-channel can be used to characterize channels with positive z.u.e. capacity. Indeed, suppose there exist two input symbols $x$ and $x^{\prime}$ and an output symbol $y$ such that $W(y \mid x)>0$ and $W\left(y \mid x^{\prime}\right)=0$. By combining all output symbols other than $y$ into a single distinct output symbol, and by using only the inputs $x$ and $x^{\prime}$, we can reduce the channel to a Z-channel with crossover probability $1-W(y \mid x)$. For this channel $C>0$, and hence also $C_{0 \text {-u }}>0$. Conversely, if $W(y \mid x)>0$ implies that $W\left(y \mid x^{\prime}\right)>0$ for all $x^{\prime} \in \mathcal{X}$, then any received sequence of output symbols can be produced by every codeword and the decoder must always erase, so $C_{0-\mathrm{u}}$ must be zero.

We also mention that z.u.e. capacity is a special case of $d$-capacity [1], and the lower bound (9) is a special case of a lower bound on $d$-capacity [1], [6], [7]. It was proved in [8] that this lower bound on $d$-capacity is tight for binaryinput channels. The proof is complicated and is, in fact, not needed when one is interested only in z.u.e. capacity. Indeed, the binary-input case is easily solved using the PinskerSheverdyaev result and the following proposition, which can also be used to improve on (6) when $|\mathcal{Y}|$ is larger than $|\mathcal{X}|+2^{|\mathcal{X}|}-1$ (see Section VI).

Proposition II.1. Suppose that the output symbols $y, y^{\prime} \in \mathcal{Y}$ are such that for every $x \in \mathcal{X}$,

$$
W(y \mid x)>0 \Longleftrightarrow W\left(y^{\prime} \mid x\right)>0 .
$$

Then the z.u.e. capacity is unaltered when we combine $y$ and $y^{\prime}$ into a single output symbol distinct from all other output symbols.

Proof: The set of messages that cannot be ruled out when $\mathbf{y}$ is observed at the output is unchanged when any occurrence of $y$ in $\mathbf{y}$ is replaced with $y^{\prime}$ or vice versa.

Using Proposition $\amalg .1$ we can reduce the output alphabet of a given DMC $W$ with input alphabet $\mathcal{X}$ to at most $2^{|\mathcal{X}|}-1$ symbols without changing the z.u.e. capacity (there are $2^{|\mathcal{X}|}-1$ possible combinations of inputs that can connect to a given output). In particular, when the input alphabet is binary, we can reduce the channel to an asymmetric binary erasure channel (possibly with some transition probabilities equal to zero). Since this channel has an acyclic bipartite channel graph, computing the z.u.e. capacity of a binary-input channel can thus be reduced to computing the Shannon capacity of an asymmetric binary erasure channel.

Unlike the Shannon capacity, the z.u.e. capacity can be increased by feedback [9]. In fact, with feedback the z.u.e. capacity $C_{0-\text { uffb }}$ is [9], [10]

$$
C_{0-\mathrm{u}, \mathrm{fb}}= \begin{cases}C & \text { if } C_{0-\mathrm{u}}>0, \\ 0 & \text { if } C_{0-\mathrm{u}}=0 .\end{cases}
$$

A concept closely related to the z.u.e. capacity is the listsize capacity [2], [11]: Suppose that instead of erasing when more than one message could have produced the observed output, the decoder instead produces a list of all messages that it cannot rule out. The listsize capacity is the supremum of all rates that are achievable in the sense that the $\rho$-th moment of the size of the list produced by the decoder tends to one as the blocklength tends to infinity; it is denoted by $C_{\mathrm{l}}(\rho)$. Here $\rho$ can be any positive number; the case $\rho=1$ has been called average-listsize capacity [2]. It is not difficult to show that $C_{\mathrm{l}}(\rho)$ never exceeds $C_{0-\mathrm{u}}$ and that $C_{\mathrm{l}}(\rho)>0$ if, and only if, $C_{0-\mathrm{u}}>0$ [11], [12]. Lower bounds on $C_{1}(\rho)$ analogous to (8) and (9) can be found in [2], [3], [11], [12]. Results for channels with feedback can be found in [12], [13], where, in particular, it is shown that feedback can increase the listsize capacity.

It was proved in [2] that Corollary I.2 is true when $C_{0 \text {-u }}$ is replaced with $C_{1}(1)$

$$
\left.\lim _{\varepsilon \rightarrow 0} C_{\mathrm{l}}(\rho)\right|_{\rho=1}=C_{\mathrm{Sp}} .
$$

This, in fact, holds for all $\rho>0$. Indeed, Theorem I.1 and the fact that $C_{1}(\rho)$ is upper-bounded by $C_{0 \text {-u }}$ for all $\rho>0$ imply that

$$
\varlimsup_{\varepsilon \rightarrow 0} C_{\mathrm{l}}(\rho) \leq C_{\mathrm{Sp}} .
$$

The reverse inequality for $0<\rho<1$ follows from (14) and the fact that $C_{1}(\rho)$ is nonincreasing in $\rho$. The case $\rho \geq 1$ is not difficult to obtain from a generalization to all $\rho>0$ of Forney's lower bound on $C_{1}(1)$ [3], [12].

It is shown in [12] that if the channel law factorizes in the sense of 11, then

$$
C_{1}(\rho)=\max _{P} \frac{E_{0}(\rho, P)}{\rho},
$$

where $E_{0}(\rho, P)$ is Gallager's function (see [14]). The RHS of (16) is also known as the cutoff rate [15]. In fact, the listsize capacity relates to the cutoff rate in much the same way that the z.u.e. capacity relates to the Shannon capacity [12], [13].

\section{A Multi-Letter Formula for $C_{0-\mathrm{u}}$}

In this section we show that (10) is asymptotically tight even when the input PMFs are restricted to be uniform over their support. 
Theorem III.1. For any DMC,

$$
C_{0-\mathrm{u}}=\lim _{n \rightarrow \infty} \frac{1}{n} \max _{P \in U_{n}} \sum_{\mathbf{y} \in \mathcal{Y}^{n}}\left(P W^{n}\right)(\mathbf{y}) \log \frac{1}{P\left(\mathcal{X}^{n}(\mathbf{y})\right)},
$$

where $U_{n}$ denotes the collection of PMFs on $\mathcal{X}^{n}$ that are uniform over their support. Moreover, the limit is equal to the supremum.

Proof: It is straightforward to verify that the sequence on the RHS of 177 without the $1 / n$ factor is superadditive, which implies that the limit is equal to the supremum 2 Let us denote this limit by $\lambda$. Achievability, i.e., $C_{0-\mathrm{u}} \geq \lambda$, follows because (10) holds for every $n$. As to the converse, let $\mathbf{x}_{1}, \ldots, \mathbf{x}_{M}$ be a codebook of blocklength $n$ and rate $R$ with maximal probability of erasure under z.u.e. decoding less than some $\delta \in(0,1)$

$$
\max _{1 \leq m \leq M} \sum_{\substack{\mathbf{y} \in \mathcal{Y}^{n}: \\ M(\mathbf{y})>1}} W^{n}\left(\mathbf{y} \mid \mathbf{x}_{m}\right)<\delta,
$$

where $M(\mathbf{y})$ denotes the number of messages that cannot be ruled out when $\mathbf{y}$ is observed at the output

$$
M(\mathbf{y})=\left|\left\{1 \leq m \leq M: W^{n}\left(\mathbf{y} \mid \mathbf{x}_{m}\right)>0\right\}\right| .
$$

Condition (18) implies that $\mathbf{x}_{m} \neq \mathbf{x}_{m^{\prime}}$ when $m \neq m^{\prime}$ because otherwise, as we next argue, the conditional probability of erasure given that the $m$-th message was sent would be one. Indeed, if $\mathbf{x}_{m}=\mathbf{x}_{m^{\prime}}$ for some $m \neq m^{\prime}$, then $M(\mathbf{y}) \geq 2$ whenever $W^{n}\left(\mathbf{y} \mid \mathbf{x}_{m}\right)>0$ (because then also $W^{n}\left(\mathbf{y} \mid \mathbf{x}_{m^{\prime}}\right)>$ $0)$, and hence

$$
\sum_{\substack{\mathbf{y} \in \mathcal{Y}^{n}: \\ M(\mathbf{y})>1}} W^{n}\left(\mathbf{y} \mid \mathbf{x}_{m}\right)=1 .
$$

Having established that the codewords are distinct, we choose $P$ to be the uniform PMF on the codebook. Then $P \in U_{n}$ and

$$
P\left(\mathcal{X}^{n}(\mathbf{y})\right)=\frac{M(\mathbf{y})}{M}, \quad \text { for all } \mathbf{y} \in \mathcal{Y}^{n} .
$$

We further observe that

$$
\begin{aligned}
\lambda & \geq \frac{1}{n} \sum_{\mathbf{y} \in \mathcal{Y}^{n}}\left(P W^{n}\right)(\mathbf{y}) \log \frac{1}{P\left(\mathcal{X}^{n}(\mathbf{y})\right)} \\
& =R-\frac{1}{n} \sum_{\substack{\mathbf{y} \in \mathcal{Y}^{n}: \\
M(\mathbf{y})>1}}\left(P W^{n}\right)(\mathbf{y}) \log M(\mathbf{y}) \\
& \geq R\left(1-\sum_{\substack{\mathbf{y} \in \mathcal{Y}^{n}: \\
M(\mathbf{y})>1}}\left(P W^{n}\right)(\mathbf{y})\right) \\
& =R\left(1-\frac{1}{M} \sum_{m=1}^{M} \sum_{\substack{\mathbf{y} \in \mathcal{Y}^{n}: \\
M(\mathbf{y})>1}} W^{n}\left(\mathbf{y} \mid \mathbf{x}_{m}\right)\right) \\
& >R(1-\delta),
\end{aligned}
$$

\footnotetext{
${ }^{2} \mathrm{~A}$ sequence $a_{1}, a_{2}, \ldots$ of real numbers is superadditive if $a_{n+m} \geq$ $a_{n}+a_{m}$ for every $n$ and $m$. For superadditive sequences $a_{n} / n$ tends to $\sup _{n} a_{n} / n$ [16 Problem 98].
}

where (22) follows because $\lambda$ is the supremum of a sequence whose $n$-th term is no smaller than the RHS of (22); where (23) follows from (21) and the fact that $\log 1=0$; where (24) follows because $M(\mathbf{y}) \leq M$; where 25) follows from the choice of $P$; and where (26) follows from (18). Thus, for any sequence of blocklength- $n$ rate- $R$ codebooks with maximal probability of erasure approaching zero,

$$
R \leq \lambda \text {. }
$$

A standard expurgation argument shows that this is also true when we replace the maximal probability of erasure with the average (over the messages) probability of erasure.

\section{Graph-Theoretic Preliminaries}

A directed graph (or simply a graph) $G$ is described by its finite vertex set $V(G)$ and its edge set $E(G) \subset V(G) \times V(G)$. We say that there is an edge from $x$ to $y$ in $G$ if $(x, y) \in E(G)$. We always assume that $G$ does not contain self-loops, i.e., that $(x, x) \notin E(G)$ for all $x \in V(G)$.

The strong product of two graphs $G$ and $H$ is denoted by $G \times H$; its vertex set is $V(G) \times V(H)$, and there is an edge from $(x, y)$ to $\left(x^{\prime}, y^{\prime}\right)$ in $G \times H$ if either $\left(x, x^{\prime}\right) \in E(G)$ and $\left(y, y^{\prime}\right) \in E(H)$, or if $\left(x, x^{\prime}\right) \in E(G)$ and $y=y^{\prime}$, or if $x=x^{\prime}$ and $\left(y, y^{\prime}\right) \in E(H)$. The $n$-fold strong product of $G$ with itself is denoted by $G^{n}$.

The subgraph of $G$ induced by $A \subseteq V(G)$ is the graph whose vertex set is $A$ and whose edge set is $E(G) \cap(A \times A)$.

A subset $A \subseteq V(G)$ is an independent set in $G$ if the subgraph of $G$ it induces has no edges, i.e., if $E(G) \cap(A \times$ $A)=\emptyset$. The maximum cardinality of an independent set in $G$ is denoted by $\alpha(G)$. We define the Sperner capacity of $G$ as

$$
\Sigma(G)=\lim _{n \rightarrow \infty} \frac{1}{n} \log \alpha\left(G^{n}\right),
$$

where the limit on the RHS is equal to the supremum because the sequence $\alpha\left(G^{1}\right), \alpha\left(G^{2}\right), \ldots$ is supermultiplicative 3 The reader is warned that this definition is not standard. However, it is equivalent to the original definition given in [17]; see Appendix C

A path in $G$ is a sequence of $n \geq 2$ distinct vertices $x_{1}, \ldots, x_{n}$ such that $\left(x_{j}, x_{j+1}\right) \in E(G)$ for all $j \in$ $\{1, \ldots, n-1\}$. The first vertex in this path is $x_{1}$, and the last vertex is $x_{n}$. We say that there is a path from $x$ to $y$ in $G$ if there is a path in $G$ whose first vertex is $x$ and whose last vertex is $y$.

A cycle is a path $x_{1}, \ldots, x_{n}$ with $\left(x_{n}, x_{1}\right) \in E(G)$. (Note that with this definition a bidirectional edge is a cycle.) We say that $G$ is acyclic if it does not contain a cycle. The maximum cardinality of a subset $A \subseteq V(G)$ that induces an acyclic subgraph of $G$ is denoted by $\rho(G)$.

The following two results will be key in the proof of Theorem I.1 The first is that $\alpha$ can be replaced with $\rho$ in (28).

Theorem IV.1. For every graph $G$,

$$
\Sigma(G)=\lim _{n \rightarrow \infty} \frac{1}{n} \log \rho\left(G^{n}\right),
$$

\footnotetext{
${ }^{3}$ A sequence $a_{1}, a_{2}, \ldots$ of real numbers is supermultiplicative if $a_{n+m} \geq$ $a_{n} a_{m}$ for all $m$ and $n$.
} 
and the limit is equal to the supremum.

In particular, Theorem IV.1 asserts that

$$
\rho\left(G^{n}\right) \leq e^{n \Sigma(G)}, \quad \text { for all } n .
$$

A proof of Theorem IV.1 is provided in Appendix A (An anonymous reviewer pointed out that a statement equivalent to Theorem IV.1 is apparently well-known; see Appendix [C)

The number of edges of $G$ ending in a vertex $x$ is called the in-degree of $x$ in $G$ and is denoted by $\mathrm{d}_{\text {in }}(x, G)$, i.e.,

$$
\mathrm{d}_{\text {in }}(x, G)=\left|\left\{x^{\prime} \in V(G):\left(x^{\prime}, x\right) \in E(G)\right\}\right| .
$$

The next result is a slight generalization of [18, p. 95, Theorem $1]$.

\section{Theorem IV.2. For every graph $G$,}

$$
\sum_{x \in V(G)} \frac{1}{1+\mathrm{d}_{\text {in }}(x, G)} \leq \rho(G) .
$$

A proof of Theorem IV.2 is provided in Appendix B.

For DMCs $W$ with $\mathcal{X} \subseteq \mathcal{Y}$ and $W(x \mid x)>0$ for every $x \in$ $\mathcal{X}$, we define the associated graph $G(W)$ to have vertex set $\mathcal{X}$ and edge set comprising all ordered pairs $(x, y)$ of distinct elements of $\mathcal{X}$ for which $W(y \mid x)>0$. Thus, for such channels we have

$$
C_{\mathrm{Sp}}(W)=\Sigma(G(W)) .
$$

Indeed, every Sperner code for $W$ of blocklength $n$ is an independent set in $G(W)^{n}$ and vice versa. We note the identity

$$
G\left(W^{n}\right)=G(W)^{n} .
$$

\section{Proof of Theorem I. 1}

Applying Jensen's Inequality to the RHS of (17) yields

$$
C_{0 \text {-u }} \leq \sup _{n \geq 1} \frac{1}{n} \max _{P \in U_{n}} \log \sum_{\mathbf{y} \in \mathfrak{S}\left(P W^{n}\right)} \frac{\left(P W^{n}\right)(\mathbf{y})}{P\left(\mathcal{X}^{n}(\mathbf{y})\right)} .
$$

It thus suffices to show that for all $P \in U_{n}$,

$$
\sum_{\mathbf{y} \in \mathfrak{S}\left(P W^{n}\right)} \frac{\left(P W^{n}\right)(\mathbf{y})}{P\left(\mathcal{X}^{n}(\mathbf{y})\right)} \leq\left(e^{C_{\mathrm{Sp}}}+\varepsilon|\mathcal{X}|(|\mathcal{Y}|-1)\right)^{n} .
$$

Fix then some $P \in U_{n}$. Since the labels do not matter, we may assume for simplicity of notation that $\mathcal{X}=\{0, \ldots,|\mathcal{X}|-1\}$ and $\mathcal{Y}=\{0, \ldots,|\mathcal{Y}|-1\}$, where $|\mathcal{Y}| \geq|\mathcal{X}|$. The distribution on $\mathcal{Y}^{n}$ induced by $P$ and $W^{n}$ can be written as

$$
\left(P W^{n}\right)(\mathbf{y})=\sum_{\substack{\mathbf{z} \in \mathcal{Y}^{n}: \\ \mathbf{y}+\mathbf{z} \in \mathcal{X}^{n}}} P(\mathbf{y}+\mathbf{z}) W^{n}(\mathbf{y} \mid \mathbf{y}+\mathbf{z}),
$$

where addition is to be understood component-wise modulo $|\mathcal{Y}|$. The $\varepsilon$-noise property (1) implies

$$
W^{n}(\mathbf{y} \mid \mathbf{y}+\mathbf{z}) \leq \varepsilon^{\|\mathbf{z}\|_{0}}, \quad \text { if } \mathbf{y}+\mathbf{z} \in \mathcal{X}^{n},
$$

where $\|\mathbf{z}\|_{0}$ denotes the number of nonzero components of $\mathbf{z}$. Thus, starting with the LHS of (36),

$$
\begin{aligned}
& \sum_{\mathbf{y} \in \mathfrak{S}\left(P W^{n}\right)} \frac{\left(P W^{n}\right)(\mathbf{y})}{P\left(\mathcal{X}^{n}(\mathbf{y})\right)} \\
& =\sum_{\mathbf{y} \in \mathfrak{S}\left(P W^{n}\right)} \sum_{\substack{\mathbf{z} \in \mathcal{Y}^{n}: \\
\mathbf{y}+\mathbf{z} \in \mathcal{X}^{n}}} \frac{P(\mathbf{y}+\mathbf{z}) W^{n}(\mathbf{y} \mid \mathbf{y}+\mathbf{z})}{P\left(\mathcal{X}^{n}(\mathbf{y})\right)} \\
& =\sum_{\mathbf{z} \in \mathcal{Y}^{n}} \sum_{\substack{\mathbf{y} \in \mathcal{Y}^{n}: \\
\mathbf{y}+\mathbf{z} \in \mathcal{X}^{n} \\
P(\mathbf{y}+\mathbf{z})>0 \\
W^{n}(\mathbf{y} \mid \mathbf{y}+\mathbf{z})>0}} \frac{P(\mathbf{y}+\mathbf{z}) W^{n}(\mathbf{y} \mid \mathbf{y}+\mathbf{z})}{P\left(\mathcal{X}^{n}(\mathbf{y})\right)}
\end{aligned}
$$

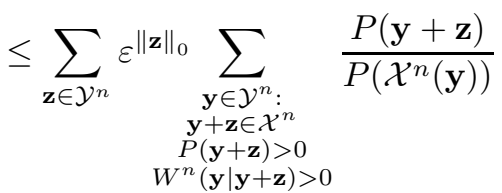

$$
\begin{aligned}
& =\sum_{\mathbf{z} \in \mathcal{Y}^{n}} \varepsilon^{\|\mathbf{z}\|_{0}} \sum_{\substack{\mathbf{y} \in \mathcal{X}^{n}: \\
P(\mathbf{y})>0 \\
W^{n}(\mathbf{y}-\mathbf{z} \mid \mathbf{y})>0}} \frac{1}{\left|\left\{\mathbf{x} \in \mathfrak{S}(P): W^{n}(\mathbf{y}-\mathbf{z} \mid \mathbf{x})>0\right\}\right|},
\end{aligned}
$$

where (40) follows from (37); where (41) follows by changing the order of summation and dropping terms that are zero; where (42) follows from (38); and where (43) follows by substituting $\mathbf{y}$ for $\mathbf{y}+\mathbf{z}$ and because $P$ is uniform over its support. For every $\mathbf{z} \in \mathcal{Y}^{n}$, let $P_{\mathbf{z}}$ be any PMF on $\mathcal{X}^{n}$ of support

$$
\mathfrak{S}\left(P_{\mathbf{z}}\right)=\left\{\mathbf{x} \in \mathcal{X}^{n}: P(\mathbf{x}) W^{n}(\mathbf{x}-\mathbf{z} \mid \mathbf{x})>0\right\} .
$$

(In fact, $P_{\mathbf{z}}$ could be any nonnegative function with the above support.) Also define for every $\mathbf{z} \in \mathcal{Y}^{n}$ the channel

$$
W_{\mathbf{z}}(\mathbf{y} \mid \mathbf{x})=W^{n}(\mathbf{y}-\mathbf{z} \mid \mathbf{x}),
$$

with input alphabet $\mathfrak{S}\left(P_{\mathbf{z}}\right)$ and output alphabet $\mathcal{Y}^{n}$. Since $\mathfrak{S}\left(P_{\mathbf{z}}\right) \subseteq \mathfrak{S}(P)$,

$$
\begin{aligned}
\left|\left\{\mathbf{x} \in \mathfrak{S}(P): W^{n}(\mathbf{y}-\mathbf{z} \mid \mathbf{x})>0\right\}\right| & \\
& \geq\left|\left\{\mathbf{x} \in \mathfrak{S}\left(P_{\mathbf{z}}\right): W_{\mathbf{z}}(\mathbf{y} \mid \mathbf{x})>0\right\}\right| .
\end{aligned}
$$

Using (46) we can upper-bound the inner sum on the RHS of (43) by

$$
\sum_{\mathbf{y} \in \mathfrak{S}\left(P_{\mathbf{z}}\right)} \frac{1}{\left\{\mathbf{x} \in \mathfrak{S}\left(P_{\mathbf{z}}\right): W_{\mathbf{z}}(\mathbf{y} \mid \mathbf{x})>0\right\} \mid} .
$$

This sum can also be written as

$$
\sum_{\mathbf{y} \in V\left(G\left(W_{\mathbf{z}}\right)\right)} \frac{1}{1+\mathrm{d}_{\text {in }}\left(\mathbf{y}, G\left(W_{\mathbf{z}}\right)\right)},
$$

where $G\left(W_{\mathbf{z}}\right)$ is the graph associated with the channel $W_{\mathbf{z}}$ (see Section IV]. Since (48) is upper-bounded by $\rho\left(G\left(W_{\mathbf{z}}\right)\right)$ (Theorem IV.2), we thus have

$$
\sum_{\mathbf{y} \in \mathfrak{S}\left(P W^{n}\right)} \frac{\left(P W^{n}\right)(\mathbf{y})}{P\left(\mathcal{X}^{n}(\mathbf{y})\right)} \leq \sum_{\mathbf{z} \in \mathcal{Y}^{n}} \varepsilon^{\|\mathbf{z}\|_{0}} \rho\left(G\left(W_{\mathbf{z}}\right)\right) .
$$

We next argue that

$$
\rho\left(G\left(W_{\mathbf{z}}\right)\right) \leq|\mathcal{X}|^{\|\mathbf{z}\|_{0}} \rho\left(G(W)^{n-\|\mathbf{z}\|_{0}}\right),
$$


where we define $\rho\left(G(W)^{0}\right)=1$. When $\|\mathbf{z}\|_{0}=n$, then (50) is trivial, so we assume that $0 \leq\|\mathbf{z}\|_{0}<n$. Let $\mathbf{x}(\mathbf{z})$ denote the restriction of $\mathbf{x} \in \mathcal{X}^{n}$ to the nonzero components of $\mathbf{z}$, and let $\mathbf{x}\left(\mathbf{z}^{\mathrm{c}}\right)$ denote the restriction of $\mathbf{x}$ to the zero components of $\mathbf{z}$. We will prove (50) by contradiction. In order to reach a contradiction, assume that for some integer $\eta$ strictly larger than the RHS of (50) there exist distinct vertices $\mathbf{x}_{1}, \ldots, \mathbf{x}_{\eta}$ in $\mathfrak{S}\left(P_{\mathbf{z}}\right)$ that induce an acyclic subgraph of $G\left(W_{\mathbf{z}}\right)$. Partition this collection of vertices by placing into the same class all $\mathbf{x}_{j}$ 's that have the same restriction $\mathbf{x}_{j}(\mathbf{z})$. Since there are $\mid \mathcal{X} \|^{\|\mathbf{z}\|_{0}}$ such classes, one of them must contain $\kappa>\rho\left(G(W)^{n-\|\mathbf{z}\|_{0}}\right)$ vertices; call them $\mathbf{x}_{1}^{\prime}, \ldots, \mathbf{x}_{\kappa}^{\prime}$. Since $\mathbf{x}_{1}^{\prime}, \ldots, \mathbf{x}_{\kappa}^{\prime}$ are distinct, and since their restrictions to the nonzero components of $\mathbf{z}$ are identical, their restrictions to the zero components of $\mathbf{z}$, i.e., $\mathbf{x}_{1}^{\prime}\left(\mathbf{z}^{\mathrm{c}}\right), \ldots, \mathbf{x}_{\kappa}^{\prime}\left(\mathbf{z}^{\mathrm{c}}\right)$ must all be distinct. Also, if $\mathbf{x}, \mathbf{y} \in \mathfrak{S}\left(P_{\mathbf{z}}\right)$ and $\mathbf{x}(\mathbf{z})=\mathbf{y}(\mathbf{z})$, then

$$
W_{\mathbf{z}}(\mathbf{y} \mid \mathbf{x})>0 \Longleftrightarrow W^{n-\|\mathbf{z}\|_{0}}\left(\mathbf{y}\left(\mathbf{z}^{\mathrm{c}}\right) \mid \mathbf{x}\left(\mathbf{z}^{\mathrm{c}}\right)\right)>0 .
$$

It follows that the subgraph of $G\left(W_{\mathbf{z}}\right)$ induced by $\mathbf{x}_{1}^{\prime}, \ldots, \mathbf{x}_{\kappa}^{\prime}$ is isomorphic to the subgraph of $G\left(W^{n-\|\mathbf{z}\|_{0}}\right)$ induced by $\mathbf{x}_{1}^{\prime}\left(\mathbf{z}^{\mathrm{c}}\right), \ldots, \mathbf{x}_{\kappa}^{\prime}\left(\mathbf{z}^{\mathrm{c}}\right){ }^{4}$ And since the former is acyclic, so must the latter be, which is a contradiction because $G\left(W^{n-\|\mathbf{z}\|_{0}}\right)=$ $G(W)^{n-\|\mathbf{z}\|_{0}}$ and $\kappa>\rho\left(G(W)^{n-\|\mathbf{z}\|_{0}}\right)$.

Having established (50), we further note that by (30) and (33),

$$
\rho\left(G(W)^{n-\|\mathbf{z}\|_{0}}\right) \leq e^{\left(n-\|\mathbf{z}\|_{0}\right) C_{\mathrm{Sp}}} .
$$

By combining (49), (50), and (52), we obtain

$$
\begin{aligned}
& \sum_{\mathbf{y} \in \mathfrak{S}\left(P W^{n}\right)} \frac{\left(P W^{n}\right)(\mathbf{y})}{P\left(\mathcal{X}^{n}(\mathbf{y})\right)} \\
& \leq \sum_{\mathbf{z} \in \mathcal{Y}^{n}} \varepsilon^{\|\mathbf{z}\|_{0}} \mid \mathcal{X} \|^{\|\mathbf{z}\|_{0}} e^{\left(n-\|\mathbf{z}\|_{0}\right) C_{\mathrm{S}_{\mathrm{p}}}} \\
& =\sum_{k=0}^{n}\left(\begin{array}{l}
n \\
k
\end{array}\right)(|\mathcal{Y}|-1)^{k} \varepsilon^{k}|\mathcal{X}|^{k} e^{(n-k) C_{\mathrm{S}_{\mathrm{p}}}},
\end{aligned}
$$

where the equality follows because the summand on the RHS of (53) depends on $\mathbf{z}$ only via $\|\mathbf{z}\|_{0}$ and there are $\left(\begin{array}{l}n \\ k\end{array}\right)(|\mathcal{Y}|-$ $1)^{k}$ elements in $\mathcal{Y}^{n}$ with exactly $k$ nonzero components. This completes the proof because the RHS of (54) is equal to the RHS of (36).

\section{CONCLUding REMARKS}

1) In Theorem $I .1$ we may replace $|\mathcal{Y}|$ with $|\mathcal{X}|+2^{|\mathcal{X}|}-1$. Indeed, using Proposition $\amalg$ II.1 and noting that the $\varepsilon$ noise property and $C_{\mathrm{Sp}}$ are preserved if we combine only output symbols in $\mathcal{Y} \backslash \mathcal{X}$, we can reduce the output alphabet to at most $|\mathcal{X}|+2^{|\mathcal{X}|}-1$ symbols.

2) The Sperner capacity of a graph and the Sperner capacity of a channel are of course just different formulations of the same problem. Indeed, in Section IV we noted that $C_{\mathrm{Sp}}(W)=\Sigma(G(W))$, where $G(W)$ is the graph associated with the $\varepsilon$-noise channel $W$. Conversely, we may associate with each directed graph $G$ a canonical

\footnotetext{
${ }^{4}$ The isomorphism is $\mathbf{x} \mapsto \mathbf{x}\left(\mathbf{z}^{\mathrm{c}}\right)$.
}

$\varepsilon$-noise channel $W_{\varepsilon}(G)$ by choosing $\mathcal{X}=\mathcal{Y}=V(G)$ and

$$
W_{\varepsilon}(G)(y \mid x)= \begin{cases}1-\varepsilon & y=x, \\ \frac{\varepsilon}{\mathrm{d}_{\text {out }}(x, G)} & (x, y) \in E(G), \\ 0 & \text { otherwise, }\end{cases}
$$

if $\mathrm{d}_{\text {out }}(x, G) \geq 1$, and otherwise

$$
W_{\varepsilon}(G)(y \mid x)= \begin{cases}1 & \text { if } y=x, \\ 0 & \text { if } y \neq x,\end{cases}
$$

where $\mathrm{d}_{\text {out }}(x, G)$ denotes the out-degree of $x$ in $G$, i.e., the number of edges of $G$ emanating from the vertex $x$. Then for any $\varepsilon \in(0,1)$,

$$
\Sigma(G)=C_{\mathrm{Sp}}\left(W_{\varepsilon}(G)\right) .
$$

3) We mentioned in the introduction that $\underline{\lim }_{\varepsilon \rightarrow 0} C_{0 \text {-u }} \geq$ $C_{\mathrm{Sp}}$ was proved in [2]. Here, we offer a simple proof based on the $n$-letter version of Forney's lower bound (10). Given $\delta>0$ choose $n$ so that there exists a Sperner code of size $e^{n\left(C_{\mathrm{s}_{\mathrm{p}}}-\delta\right)}$. Let $P$ be the uniform PMF on the codebook and note that

$$
\left(P W^{n}\right)(\mathbf{x}) \geq P(\mathbf{x})(1-\varepsilon)^{n} \quad \text { for all } \mathbf{x} \in \mathcal{X}^{n} .
$$

For this $P$,

$$
\begin{aligned}
C_{0-\mathrm{u}} & \geq \frac{1}{n} \sum_{\mathbf{y} \in \mathcal{Y}^{n}}\left(P W^{n}\right)(\mathbf{y}) \log \frac{1}{P\left(\mathcal{X}^{n}(\mathbf{y})\right)} \\
& \geq \frac{1}{n} \sum_{m=1}^{e^{n\left(C_{\mathrm{S}_{\mathrm{p}}}-\delta\right)}}\left(P W^{n}\right)\left(\mathbf{x}_{m}\right) \log \frac{1}{P\left(\mathcal{X}^{n}\left(\mathbf{x}_{m}\right)\right)} \\
& =\frac{1}{n} \sum_{m=1}^{e^{n\left(C_{\mathrm{S}_{\mathrm{p}}}-\delta\right)}}\left(P W^{n}\right)\left(\mathbf{x}_{m}\right) \log \frac{1}{P\left(\mathbf{x}_{m}\right)} \\
& \geq(1-\varepsilon)^{n} \frac{1}{n} \sum_{m=1}^{e^{n\left(C_{\mathrm{Sp}_{\mathrm{p}}}-\delta\right)}} P\left(\mathbf{x}_{m}\right) \log \frac{1}{P\left(\mathbf{x}_{m}\right)} \\
& =(1-\varepsilon)^{n}\left(C_{\mathrm{Sp}}-\delta\right),
\end{aligned}
$$

where (59) follows from (10); where (60) follows because the summand is nonnegative; where (61) follows from the choice of $P$ and the property of Sperner codes (4); where (62) follows from (58); and where (63) follows from the choice of $P$. Letting first $\varepsilon \rightarrow 0$ and then $\delta \rightarrow 0$ completes the proof.

4) For some channels the bound in Theorem I.1 can be sharpened. For example, for the canonical $\varepsilon$-noise channel associated with the cyclic orientation of a triangle $C_{\mathrm{Sp}}=\log 2$ [19], [20], and it is shown in [21] that $C_{0 \text {-u }} \leq \log 2$ for every $\varepsilon \in(0,1)$.

\section{APPENDIX A \\ Proof of THEOREM IV.1}

We shall need the elementary fact that the vertices of any acyclic graph $G$ can be labeled with the numbers $1, \ldots,|V(G)|$ such that $(x, y) \in E(G)$ only if $x<y$ (see, e.g., [22, Section 5.7]) 5

\footnotetext{
${ }^{5}$ A different way to state this is that any partial order on a finite set can be extended to a total order on this set.
} 
Using this fact, we first show that the sequence $\rho\left(G^{1}\right), \rho\left(G^{2}\right), \ldots$ is supermultiplicative, which will imply that the limit on the RHS of (29) equals the supremum. Choose for each $n$ some $A_{n} \subseteq V(G)^{n}$ that achieves $\rho\left(G^{n}\right)$, i.e., $A_{n}$ induces an acyclic subgraph of $G^{n}$ and $\left|A_{n}\right|=\rho\left(G^{n}\right)$. We show that $A_{n} \times A_{m}$ induces an acyclic subgraph of $G^{n+m}$ and hence that

$$
\begin{aligned}
\rho\left(G^{n+m}\right) & \geq\left|A_{n} \times A_{m}\right| \\
& =\rho\left(G^{n}\right) \rho\left(G^{m}\right) .
\end{aligned}
$$

Label the vertices in $A_{n}$ with the numbers $1, \ldots,\left|A_{n}\right|$ so that $\left(x, x^{\prime}\right) \in E\left(G^{n}\right) \cap\left(A_{n} \times A_{n}\right)$ implies $x<x^{\prime}$. Similarly label the vertices in $A_{m}$. To reach a contradiction, assume that $\left(x_{1}, y_{1}\right), \ldots,\left(x_{\eta}, y_{\eta}\right)$ is a cycle in the subgraph of $G^{n+m}$ induced by $A_{n} \times A_{m}$. From the definition of strong product and the labeling of the vertices it follows that $x_{1}<x_{\eta}$ or $y_{1}<y_{\eta}$. Consequently, there cannot be an edge from $\left(x_{\eta}, y_{\eta}\right)$ to $\left(x_{1}, y_{1}\right)$ in this subgraph, which contradicts the assumption that $\left(x_{1}, y_{1}\right), \ldots,\left(x_{\eta}, y_{\eta}\right)$ is a cycle.

As to 29), we first show that

$$
\Sigma(G)=\log |V(G)|, \quad \text { for all acyclic } G .
$$

Note that this will prove Theorem IV.1 in the special case where $G$ is acyclic. Indeed, in this case $\rho(G)=|V(G)|$, so (65) implies $\rho\left(G^{n}\right) \geq|V(G)|^{n}$. And since clearly $\rho\left(G^{n}\right) \leq$ $|V(G)|^{n}$, we thus have

$$
\rho\left(G^{n}\right)=|V(G)|^{n}, \quad \text { for all acyclic } G .
$$

To prove (66), note that $\alpha\left(G^{n}\right) \leq|V(G)|^{n}$ and hence $\Sigma(G) \leq$ $\log |V(G)|$ (this is true for any $G$, not just acyclic), so it only remains to prove the reverse inequality. Since $G$ is acyclic, we may label its vertices with the numbers $1, \ldots,|V(G)|$ so that there is an edge from $x$ to $y$ in $G$ only if $x<y$. We then define the weight of a vertex $\mathbf{x}$ in $G^{n}$ as the sum of the labels of its $n$ components. Thus, the weight is a number between $n$ and $n|V(G)|$.

As we next show, if $A$ is a subset of $V(G)^{n}$ all of whose members have the same weight, then $A$ is an independent set in $G^{n}$. Indeed, if $\mathbf{x}$ and $\mathbf{y}$ are distinct vertices in $A$, then $x^{(j)}>$ $y^{(j)}$, say, for some $j \in\{1, \ldots, n\}$. Since $\mathbf{x}$ and $\mathbf{y}$ have equal weight, there must also be some $k \neq j$ for which $x^{(k)}<y^{(k)}$. Thus, $\left(x^{(j)}, y^{(j)}\right) \notin E(G)$ and $\left(y^{(k)}, x^{(k)}\right) \notin E(G)$, so there is no edge from $\mathbf{x}$ to $\mathbf{y}$ and no edge from $\mathbf{y}$ to $\mathbf{x}$ in $G^{n}$.

If we partition $V(G)^{n}$ by putting in the same class all vertices of the same weight, then one of the $n|V(G)|-n+1$ different classes must have at least

$$
\frac{|V(G)|^{n}}{n|V(G)|-n+1}
$$

members. Thus,

$$
\frac{1}{n} \log \alpha\left(G^{n}\right) \geq \log |V(G)|-\frac{1}{n} \log (n|V(G)|-n+1),
$$

and letting $n$ tend to infinity establishes $\Sigma(G) \geq \log |V(G)|$ and hence proves (66).

To complete the proof of 29), let $G$ be any graph (not necessarily acyclic) and let $\lambda$ denote the limit of $n^{-1} \log \rho\left(G^{n}\right)$ as $n$ tends to infinity (i.e., the supremum). For a given $\delta>0$ select $\nu$ so that

$$
\frac{1}{\nu} \log \rho\left(G^{\nu}\right) \geq \lambda-\delta .
$$

Choose $A \subseteq V(G)^{\nu}$ that achieves $\rho\left(G^{\nu}\right)$ and let $H$ denote the acyclic subgraph of $G^{\nu}$ it induces. Since $H^{m}$ is the subgraph of $G^{\nu m}$ induced by $A^{m}$,

$$
\frac{1}{\nu m} \log \alpha\left(G^{\nu m}\right) \geq \frac{1}{\nu m} \log \alpha\left(H^{m}\right) .
$$

Letting $m$ tend to infinity, we obtain

$$
\Sigma(G) \geq \frac{1}{\nu} \Sigma(H)
$$

Since $H$ is acyclic, we can substitute it for $G$ in 66 to obtain

$$
\begin{aligned}
\frac{1}{\nu} \Sigma(H) & =\frac{1}{\nu} \log |A| \\
& =\frac{1}{\nu} \log \rho\left(G^{\nu}\right),
\end{aligned}
$$

where (74) follows because $A$ achieves $\rho\left(G^{\nu}\right)$. Combining (72), (74), and (70) shows that $\Sigma(G) \geq \lambda-\delta$. Since this is true for every $\delta>0$,

$$
\Sigma(G) \geq \lambda
$$

On the other hand, a graph with no edges is trivially acyclic, so $\alpha\left(G^{n}\right) \leq \rho\left(G^{n}\right)$ and hence $\Sigma(G) \leq \lambda$.

\section{APPENDIX B \\ PROOF OF THEOREM IV.2}

Let $<$ be a total ordering of the vertices of $G$ and consider the subset $A \subseteq V(G)$ comprising all $x \in V(G)$ such that if $\left(x^{\prime}, x\right) \in E(G)$ for some $x^{\prime} \in V(G)$, then $x^{\prime}<x$. The subgraph of $G$ induced by $A$ is acyclic. Indeed, if $x_{1}, \ldots, x_{\eta}$ is a path in this subgraph, then $x_{1}<x_{\eta}$, so we cannot have $\left(x_{\eta}, x_{1}\right) \in E(G)$. Thus,

$$
|A| \leq \rho(G) .
$$

Suppose now that $<$ is drawn uniformly at random among all total orderings of $V(G)$. Then

$$
\operatorname{Pr}(x \in A)=\frac{1}{1+\mathrm{d}_{\text {in }}(x, G)}, \quad \text { for all } x \in V(G) .
$$

Indeed, $x$ is in $A$ if, and only if, it is the greatest vertex in the set

$$
B=\{x\} \cup\left\{x^{\prime}:\left(x^{\prime}, x\right) \in E(G)\right\} .
$$

Since $<$ is drawn uniformly at random, every vertex in $B$ has the same probability of being the greatest element in $B$, so (77) follows by noting that $|B|=1+\mathrm{d}_{\text {in }}(x, G)$.

Summing both sides of (77) over all vertices of $G$ yields

$$
\sum_{x \in V(G)} \frac{1}{1+\mathrm{d}_{\text {in }}(x, G)}=\sum_{x \in V(G)} \operatorname{Pr}(x \in A) .
$$

By writing $\operatorname{Pr}(x \in A)$ as the expectation of the indicator function of the event $\{x \in A\}$ and by swapping summation and expectation, we see that the RHS of (79) is the expected cardinality of $A$. This expected cardinality cannot exceed $\rho(G)$ because (76) holds for every realization of $<$. 


\section{APPENDIX C}

REMARKS ABOUt THE DEFINITION OF SPERnER CAPACITY

It was pointed out in Section IV that the definition of Sperner capacity used in this paper (28) is not standard. Here we compare this definition to the original one given in [17] and discuss their equivalence. But first, we need some more notation.

The weak product of two directed graphs $G$ and $H$ is denoted by $G \cdot H$. The vertex set of $G \cdot H$ is $V(G) \times V(H)$ and there is an edge from $(x, y)$ to $\left(x^{\prime}, y^{\prime}\right)$ in $G \cdot H$ if $\left(x, x^{\prime}\right) \in E(G)$ or $\left(y, y^{\prime}\right) \in E(H)$. The $n$-fold weak product of $G$ with itself is denoted by $G^{\vee n}$.

The complement $G^{c}$ of a directed graph $G$ has vertex set $V\left(G^{c}\right)=V(G)$ and edge set

$E\left(G^{c}\right)=(V(G) \times V(G)) \backslash(E(G) \cup\{(x, x): x \in V(G)\})$,

i.e., $E\left(G^{c}\right)$ is the set complement of $E(G)$ in $V(G) \times V(G)$ minus the self-loops. Note that $\left(G^{c}\right)^{c}=G$.

A subset $A \subseteq V(G)$ is a symmetric clique in $G$ if the subgraph it induces is complete, i.e., if $(x, y) \in E(G)$ whenever $x$ and $y$ are distinct vertices in $A$. The largest cardinality of a symmetric clique in $G$ is denoted by $\omega(G)$. In [17], the Sperner capacity of $G$ is defined as

$$
\Sigma_{0}(G)=\lim _{n \rightarrow \infty} \frac{1}{n} \log \omega\left(G^{\vee n}\right) .
$$

(We use $\Sigma_{0}$ instead of $\Sigma$ to distinguish it from our nonstandard definition (28).) This definition is equivalent to the definition (28) in the following sense: For every directed graph $G$,

$$
\Sigma(G)=\Sigma_{0}\left(G^{c}\right)
$$

To prove this relationship, first note that an independent set in $G$ is a symmetric clique in $G^{c}$ and vice versa. Hence $\alpha(G)=\omega\left(G^{c}\right)$. Moreover, one easily verifies that $\left(G^{n}\right)^{c}=$ $\left(G^{c}\right)^{\vee n}$. Consequently,

$$
\begin{aligned}
\alpha\left(G^{n}\right) & =\omega\left(\left(G^{n}\right)^{c}\right) \\
& =\omega\left(\left(G^{c}\right)^{\vee n}\right),
\end{aligned}
$$

and 81 follows.

The reason we prefer (28) over (80) is Theorems IV.2 and IV.1.

An anonymous reviewer pointed out to us that an equivalent version of Theorem IV.1 is well-known. To state it, we need the concept of transitive cliques:

A subset of vertices $A \subseteq V(G)$ is called a transitive clique in $G$ if there exists a total order $<$ on $A$ with the property

$$
(x, y \in A \text { and } x<y) \Longrightarrow(x, y) \in E(G) \text {. }
$$

It is easily verified that a subset $A \subseteq V(G)$ induces an acyclic subgraph of $G$ if, and only if, $A$ is a transitive clique in $G^{c}$. Thus, if $\omega_{\text {tr }}(G)$ denotes the size of the largest transitive clique in $G$, then

$$
\rho(G)=\omega_{\mathrm{tr}}\left(G^{c}\right) .
$$

Hence, Theorem IV.1 is equivalent to the statement that

$$
\Sigma_{0}(G)=\lim _{n \rightarrow \infty} \frac{1}{n} \log \omega_{\mathrm{tr}}\left(G^{\vee n}\right),
$$

i.e., that $\omega$ can be replaced with $\omega_{\text {tr }}$ in the original definition of Sperner capacity (80). Although we could not find a reference where this is explicitly proven, it was certainly used in [23].

\section{ACKNOWLEDGMENT}

We are grateful for valuable comments and suggestions from the anonymous reviewers and the Associate Editor.

\section{REFERENCES}

[1] I. Csiszár and P. Narayan, "Channel capacity for a given decoding metric," IEEE Trans. Inf. Theory, vol. 41, no. 1, pp. 35-43, 1995.

[2] R. Ahlswede, N. Cai, and Z. Zhang, "Erasure, list, and detection zeroerror capacities for low noise and a relation to identification," IEEE Trans. Inf. Theory, vol. 42, no. 1, pp. 55-62, 1996.

[3] G. Forney Jr, "Exponential error bounds for erasure, list, and decision feedback schemes," IEEE Trans. Inf. Theory, vol. 14, no. 2, pp. 206-220, 1968.

[4] İ. E. Telatar, "Multi-access communications with decision feedback decoding," Ph.D. dissertation, Massachusetts Institute of Technology, Dept. of Electrical Engineering and Computer Science, May 1992.

[5] M. S. Pinsker and A. Y. Sheverdyaev, "Transmission capacity with zero error and erasure," Problemy Peredachi Informatsii, vol. 6, no. 1, pp. 20-24, 1970.

[6] J. Y. N. Hui, "Fundamental issues of multiple accessing," Ph.D. dissertation, Massachusetts Institute of Technology, Dept. of Electrical Engineering and Computer Science, November 1983.

[7] I. Csiszár and J. Körner, "Graph decomposition: A new key to coding theorems," IEEE Trans. Inf. Theory, vol. 27, no. 1, pp. 5-12, 1981.

[8] V. B. Balakirsky, "A converse coding theorem for mismatched decoding at the output of binary-input memoryless channels," IEEE Trans. Inf. Theory, vol. 41, no. 6, pp. 1889-1902, 1995.

[9] C. Bunte and A. Lapidoth, "The zero-undetected-error capacity of discrete memoryless channels with feedback," in Communication, Control, and Computing (Allerton), 2012 50th Annual Allerton Conference on. IEEE, 2012, pp. 1838-1842.

[10] B. Nakiboğlu and L. Zheng, "Errors-and-erasures decoding for block codes with feedback," IEEE Trans. Inf. Theory, vol. 58, no. 1, pp. 2449, 2012.

[11] İ. E. Telatar, "Zero-error list capacities of discrete memoryless channels," IEEE Trans. Inf. Theory, vol. 43, no. 6, pp. 1977-1982, 1997.

[12] C. Bunte and A. Lapidoth, "On the listsize capacity with feedback," submitted to the IEEE Transactions on Information Theory, Nov. 2013, http://arxiv.org/abs/1311.0195

[13] C. Bunte and A. Lapidoth, "On the cutoff rate and the average-listsize capacity of discrete memoryless channels with feedback," in Information Theory Workshop (ITW), 2013 IEEE, 2013, pp. 649-653.

[14] R. G. Gallager, Information Theory and Reliable Communication. New York: John Wiley \& Sons, 1968.

[15] E. Arıkan, "An inequality on guessing and its application to sequential decoding," IEEE Trans. Inf. Theory, vol. 42, no. 1, pp. 99-105, 1996.

[16] G. Pólya and G. Szegó, Problems and Theorems in Analysis I. Berlin Heidelberg: Springer-Verlag, 1978.

[17] L. Gargano, J. Körner, and U. Vaccaro, "Qualitative independence and Sperner problems for directed graphs," Journal of Combinatorial Theory, Series A, vol. 61, no. 2, pp. 173-192, 1992.

[18] N. Alon and J. H. Spencer, The Probabilistic Method, 3rd ed. Hoboken, NJ: Wiley, 2008.

[19] A. R. Calderbank, P. Frankl, R. L. Graham, W.-C. W. Li, and L. A. Shepp, "The Sperner capacity of linear and nonlinear codes for the cyclic triangle," Journal of Algebraic Combinatorics, vol. 2, no. 1, pp. 31-48, 1993.

[20] A. Blokhuis, "On the Sperner capacity of the cyclic triangle," Journal of Algebraic Combinatorics, vol. 2, no. 2, pp. 123-124, 1993.

[21] C. Bunte, A. Lapidoth, and A. Samorodnitsky, "The zero-undetectederror capacity of the low-noise cyclic triangle channel," in Information Theory Proceedings (ISIT), 2013 IEEE International Symposium on, 2013, pp. 91-95.

[22] K. Thulasiraman and M. N. S. Swamy, Graphs: Theory and Algorithms. New York: John Wiley \& Sons, 1992.

[23] J. Körner, "On clique growth in products of directed graphs," Graphs and Combinatorics, vol. 14, no. 1, pp. 25-36, 1998. 